\title{
Constant Modulus Algorithm Aided Soft Decision-Directed Blind Space-Time Equalization for SIMO Channels
}

\author{
S. Chen, A. Wolfgang and L. Hanzo \\ School of Electronics and Computer Science \\ University of Southampton, Southampton SO17 1BJ, U.K.
}

\begin{abstract}
Smart antenna aided broadband beamforming plays an increasingly important role in wireless communications. The paper investigates blind space-time equalization/equalizers (STE) designed for single-input multi-output (SIMO) systems. Specifically, the constant modulus algorithm (CMA) and a soft decision-directed (SDD) scheme, originally derived for low-complexity blind equalization of single-input single-output (SISO) channels, are combined for employment in the SIMO scenario.
\end{abstract}

\section{INTRODUCTION}

Space-time processing techniques have increasingly been applied to wireless communication systems [1]-[6]. With the aid of smart antenna arrays and by exploiting both the space and time dimensions, space-time processing is capable of effectively improving the system's capacity, coverage and quality by suppressing intersymbol interference and reducing co-channel interference. In this paper, we investigate a blind STE designed for the SIMO channel by employing a transmitter using a single antenna and a receiver employing multiple antennas. We extend the combined CMA SDD scheme, originally derived for SISO systems [7], for employment in SIMO system. Simulations are conducted to compare the performance of this blind adaptive scheme to that of a CMA and decision directed (DD) scheme, which was originally derived also for the SISO system [8].

\section{SySTEM MODEL}

Consider the SIMO system employing $L$ receiver antennas, as depicted in Fig. 1, where $s(t)$ is the transmitted signal, $x_{l}(t)$ denotes the $l$ th antenna's output signal and $e_{l}(t)$ the $l$ th channel's noise. The received signals are sampled at a rate of twice the symbol rate $f_{s}$ to obtain $\bar{x}_{l}(n), 1 \leq l \leq L$, which are passed to the STE shown in Fig. 2. Since the sampling rate is twice $f_{s}$, the delay unit $\Delta$ used in the temporal filters equals $T_{s} / 2$, where $T_{s}$ is the symbol period. For

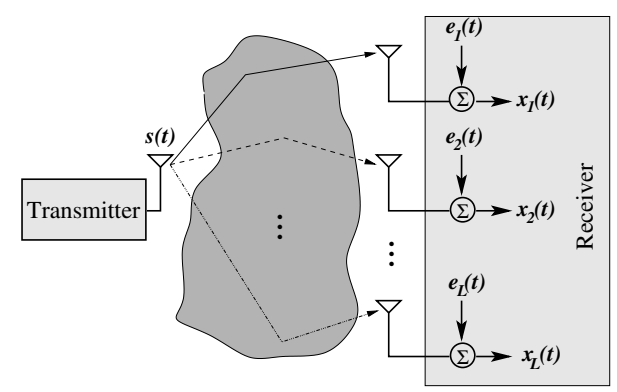

Fig. 1. Single-input multiple-output system employing multiple receiver antennas. notational convenience, the index $k$ is reserved for $T_{s}$-spaced sampled quantities and the index $n$ for $T_{s} / 2$-spaced sampled quantities throughout our discussions. The transmitted $T_{s}$-spaced complexvalued symbol sequence $s(k)=s_{R}(k)+j s_{I}(k)$ is assumed to be i.i.d. and the symbol constellation is $M$-QAM with the set of all the phasor points defined by

$$
\mathcal{S}=\left\{s_{i, l}=(2 i-Q-1)+j(2 l-Q-1), 1 \leq i, l \leq Q\right\},
$$

where we have $Q=\sqrt{M}$ and $j=\sqrt{-1}$. The $T_{s} / 2$-spaced signal samples $\bar{x}_{l}(n)$ of the $l$ th antenna are given by

$$
\bar{x}_{l}(n)=\sum_{i=0}^{2 N_{c}-1} \bar{a}_{i, l} \bar{s}(n-i)+\bar{e}_{l}(n),
$$

where the $T_{s} / 2$-spaced sequence $\{\bar{s}(n)\}$ is a zero-padded version of the transmitted symbol sequence $\{s(k)\}$ defined by $\bar{s}(n)=$ $s(n / 2)$ for even $n$ and $\bar{s}(n)=0$ for odd $n$. Furthermore the $T_{s} / 2$-spaced complex-valued Channel Impulse Response (CIR) of the $l$ th channel is given by $\overline{\mathbf{a}}_{l}=\left[\begin{array}{llll}\bar{a}_{0, l} & \bar{a}_{1, l} & \bar{a}_{2, l} & \bar{a}_{3, l} \cdots \bar{a}_{2 N_{c}-1, l}\end{array}\right]^{T}$, with $N_{c}$ corresponding to the $T_{s}$-spaced $l$ th CIR duration, and the $T_{s} / 2$-spaced noise sample $\bar{e}_{l}(n)=\bar{e}_{l, R}(n)+j \bar{e}_{l, I}(n)$ is an i.i.d. complex-valued Gaussian white noise process associated with $\mathrm{E}\left[\bar{e}_{l, R}^{2}(n)\right]=\mathrm{E}\left[\bar{e}_{l, I}^{2}(n)\right]=\sigma_{e}^{2}$.

The STE consists of $L$ number of $T_{s} / 2$-spaced temporal filters, and its output is defined by

$$
\bar{y}(n)=\sum_{l=1}^{L} \sum_{i=0}^{2 m-1} \bar{w}_{i, l} \bar{x}_{l}(n-i)=\sum_{l=1}^{L} \overline{\mathbf{w}}_{l}^{T} \overline{\mathbf{x}}_{l}(n)=\overline{\mathbf{w}}^{T} \overline{\mathbf{x}}(n)
$$

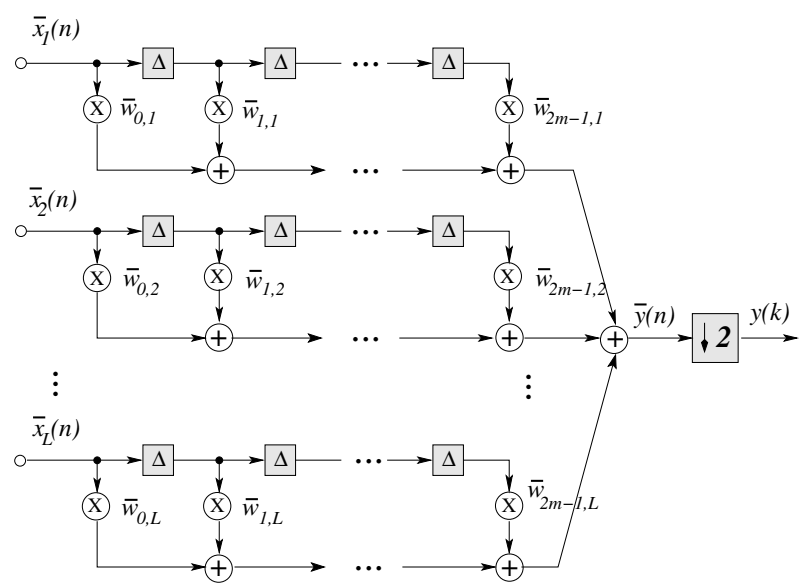

Fig. 2. Space-time equalization structure using $\Delta$-spaced temporal filters, where $\Delta=$ $T_{s} / 2$ and $T_{s}$ denotes the symbol period, the index $k$ indicates $T_{s}$-spaced sampled quantities and the index $n T_{s} / 2$-spaced sampled quantities. 
where $2 m$ is the order of the $T_{s} / 2$-spaced temporal filters, $\overline{\mathbf{w}}=$ $\left[\overline{\mathbf{w}}_{1}^{T} \overline{\mathbf{w}}_{2}^{T} \cdots \overline{\mathbf{w}}_{L}^{T}\right]^{T}$ and $\overline{\mathbf{x}}(n)=\left[\overline{\mathbf{x}}_{1}^{T}(n) \overline{\mathbf{x}}_{2}^{T}(n) \cdots \overline{\mathbf{x}}_{L}^{T}(n)\right]^{T}$, with $\overline{\mathbf{w}}_{l}=\left[\begin{array}{llll}\bar{w}_{0, l} & \bar{w}_{1, l} \cdots \bar{w}_{2 m-1, l}\end{array}\right]^{T}$ and $\overline{\mathbf{x}}_{l}(n)=\left[\bar{x}_{l}(n) \bar{x}_{l}(n-\right.$ 1) $\left.\cdots \bar{x}_{l}(n-2 m+1)\right]^{T}$ being the complex-valued weight vector and input vector of the $l$ th temporal filter, respectively. The $T_{s} / 2$-spaced output $\bar{y}(n)$ is decimated by a factor of 2 to create the $T_{s}$-spaced output $y(k)$. Following the same approach for the SISO fractionally spaced equalizer [9], the $T_{s}$-spaced model of $y(k)$ can be derived, which can be used for developing adaptive algorithms. Let us first define the even and odd channels of $\overline{\mathbf{a}}_{l}$ as $\overline{\mathbf{a}}_{l}^{\mathrm{e}}=$ $\left[\begin{array}{ll}\bar{a}_{0, l} & \bar{a}_{2, l} \cdots \bar{a}_{2 N_{c}-2, l}\end{array}\right]^{T}$ and $\overline{\mathbf{a}}_{l}^{\mathrm{o}}=\left[\bar{a}_{1, l} \bar{a}_{3, l} \cdots \bar{a}_{2 N_{c}-1, l}\right]^{T}$, the even and odd equalizers of $\overline{\mathbf{w}}_{l}$ as $\overline{\mathbf{w}}_{l}^{\mathrm{e}}=\left[\begin{array}{lll}\bar{w}_{0, l} & \bar{w}_{2, l} \cdots \bar{w}_{2 m-2, l}\end{array}\right]^{T}$ and $\overline{\mathbf{w}}_{l}^{\mathrm{o}}=\left[\bar{w}_{1, l} \bar{w}_{3, l} \cdots \bar{w}_{2 m-1, l}\right]^{T}$, the even and odd noise samples as $e_{l}^{\mathrm{e}}(k)=\bar{e}_{l}(2 n)$ and $e_{l}^{\mathrm{o}}(k)=\bar{e}_{l}(2 n+1)$, and the even and odd samples of $x_{l}(n)$ as $x_{l}^{\mathrm{e}}(k)=\bar{x}_{l}(2 n)$ and $x_{l}^{\mathrm{o}}(k)=\bar{x}_{l}(2 n+1)$. Furthermore we define

$$
\mathbf{w}_{l}=\left[\begin{array}{llll}
w_{0, l} & w_{1, l} \cdots w_{2 m-1, l}
\end{array}\right]^{T}=\left[\begin{array}{ll}
\left(\overline{\mathbf{w}}_{l}^{\mathrm{o}}\right)^{T} & \left(\overline{\mathbf{w}}_{l}^{\mathrm{e}}\right)^{T}
\end{array}\right]^{T}
$$

and

$$
\begin{gathered}
\mathbf{x}_{l}(k)=\left[x_{l}(k) x_{l}(k-1) \cdots x_{l}(k-2 m+1)\right]^{T} \\
=\left[\left(\mathbf{x}_{l}^{\mathrm{e}}(k)\right)^{T}\left(\mathbf{x}_{l}^{\mathrm{o}}(k)\right)^{T}\right]^{T}
\end{gathered}
$$

with $\mathbf{x}_{l}^{\mathrm{e}}(k)=\left[x_{l}^{\mathrm{e}}(k) x_{l}^{\mathrm{e}}(k-1) \cdots x_{l}^{\mathrm{e}}(k-m+1)\right]^{T}$ and $\mathbf{x}_{l}^{\mathrm{o}}(k)=$ $\left[x_{l}^{\mathrm{o}}(k) x_{l}^{\mathrm{o}}(k-1) \cdots x_{l}^{\mathrm{o}}(k-m+1)\right]^{T}$. Then the $T_{s}$-spaced STE output $y(k)$ is given by

$$
y(k)=\sum_{l=1}^{L} \sum_{i=0}^{2 m-1} w_{i, l} x_{l}(k-i)=\sum_{l=1}^{L} \mathbf{w}_{l}^{T} \mathbf{x}_{l}(k)=\mathbf{w}^{T} \mathbf{x}(k)
$$

with $\mathbf{w}=\left[\mathbf{w}_{1}^{T} \cdots \mathbf{w}_{L}^{T}\right]^{T}$ and $\mathbf{x}(k)=\left[\mathbf{x}_{1}^{T}(k) \cdots \mathbf{x}_{L}^{T}(k)\right]^{T}$. The overall signal to noise ratio (SNR) of the system is defined as

$$
\mathrm{SNR}=\frac{\sigma_{s}^{2} \sum_{l=1}^{L} \sum_{i=0}^{2 N_{c}-1}\left|\bar{a}_{i, l}\right|^{2}}{4 L \sigma_{e}^{2}}
$$

where $\sigma_{s}^{2}=E\left[|s(k)|^{2}\right]$. We will extend an initialization strategy commonly used for SISO blind equalization [9] to the present blind STE. Before blind adaptation, the equalizer weights are initialized to $\bar{w}_{i, l}=\frac{1}{L}+j 0$ for $i=m-1$ and $m$, and $\bar{w}_{i, l}=0+j 0$ for all the other values of $i$, where $1 \leq l \leq L$.

\section{CMA Aided Soft Decision DiRected Scheme}

Let each temporal filter of the STE be split into two parts, namely $\mathbf{w}_{l}=\mathbf{w}_{c_{l}}+\mathbf{w}_{d_{l}}$, and denote $\mathbf{w}=\mathbf{w}_{c}+\mathbf{w}_{d}$. The weight vector $\mathbf{w}_{c}$ of the CMA STE is designed for minimizing the CMA's cost function

$$
\bar{J}_{\mathrm{CMA}}(\mathbf{w})=\mathrm{E}\left[\left(|y(k)|^{2}-R^{2}\right)^{2}\right]
$$

by adapting $\mathbf{w}_{c}$ using a stochastic gradient algorithm, where $R^{2}=$ $\mathrm{E}\left[|s(k)|^{4}\right] / \mathrm{E}\left[|s(k)|^{2}\right]$. Similar to the case of SISO blind equalization [10][11], at the $T_{s}$-spaced sample $k$ and given $y(k)=$ $\mathbf{w}^{T}(k) \mathbf{x}(k)$, the CMA adapts $\mathbf{w}_{c}$ according to

$$
\left.\begin{array}{l}
\epsilon(k)=y(k)\left(R^{2}-|y(k)|^{2}\right) \\
\mathbf{w}_{c}(k+1)=\mathbf{w}_{c}(k)+\mu_{\mathrm{CMA}} \epsilon(k) \mathbf{x}^{*}(k)
\end{array}\right\},
$$

where $\mu_{\mathrm{CMA}}$ is a small positive adaptive gain factor and $\mathbf{x}^{*}(k)$ is the complex conjugate of $\mathbf{x}(k)$. Typically, a small adaptive gain $\mu_{\mathrm{CMA}}$ has to be used for ensuring convergence.

Let the complex plane be divided into $M / 4$ rectangular regions, as illustrated in Fig. 3. Each region $S_{i, l}$ contains four symbol points

$$
S_{i, l}=\left\{s_{p, q}, p=2 i-1,2 i, q=2 l-1,2 l\right\} .
$$

If the equalizer output $y(k)$ is within the region $S_{i, l}$, a local approximation to the a posteriori probability density function (p.d.f.) of $y(k)$ is [13]

$$
\hat{p}(\mathbf{w}, y(k)) \approx \sum_{p=2 i-1}^{2 i} \sum_{q=2 l-1}^{2 l} \frac{1}{8 \pi \rho} \exp \left(-\frac{\left|y(k)-s_{p, q}\right|^{2}}{2 \rho}\right),
$$

where $\rho$ is the "variance" or width of the clusters. This approximation is only valid however when the equalizer has converged. Before reaching this stage, a bootstrap optimization process can be performed for arriving at the MAP solution, as presented in [12],[13]. The subsequently activated SDD equalizer was designed for maximizing the log of the local a posteriori p.d.f. criterion was

$$
\bar{J}_{\text {LMAP }}(\mathbf{w})=\mathrm{E}\left[J_{\text {LMAP }}(\mathbf{w}, y(k))\right]
$$

by adjusting $\mathbf{w}_{d}$ using a stochastic gradient algorithm, where

$$
J_{\text {LMAP }}(\mathbf{w}, y(k))=\rho \log (\hat{p}(\mathbf{w}, y(k))) .
$$

Specifically, the SDD equalizer adapts $\mathbf{w}_{d}$ according to

$$
\mathbf{w}_{d}(k+1)=\mathbf{w}_{d}(k)+\mu_{\mathrm{SDD}} \frac{\partial J_{\mathrm{LMAP}}(\mathbf{w}(k), y(k))}{\partial \mathbf{w}_{d}}
$$

where we have

$$
\begin{gathered}
\frac{\partial J_{\mathrm{LMAP}}(\mathbf{w}, y(k))}{\partial \mathbf{w}_{d}}= \\
\frac{\sum_{p=2 i-1}^{2 i} \sum_{q=2 l-1}^{2 l} \exp \left(-\frac{\left|y(k)-s_{p, q}\right|^{2}}{2 \rho}\right)\left(s_{p, q}-y(k)\right)}{\sum_{p=2 i-1}^{2 i} \sum_{q=2 l-1}^{2 l} \exp \left(-\frac{\left|y(k)-s_{p, q}\right|^{2}}{2 \rho}\right)} \mathbf{x}^{*}(k)
\end{gathered}
$$

and $\mu_{\mathrm{SDD}}$ is an adaptive gain.

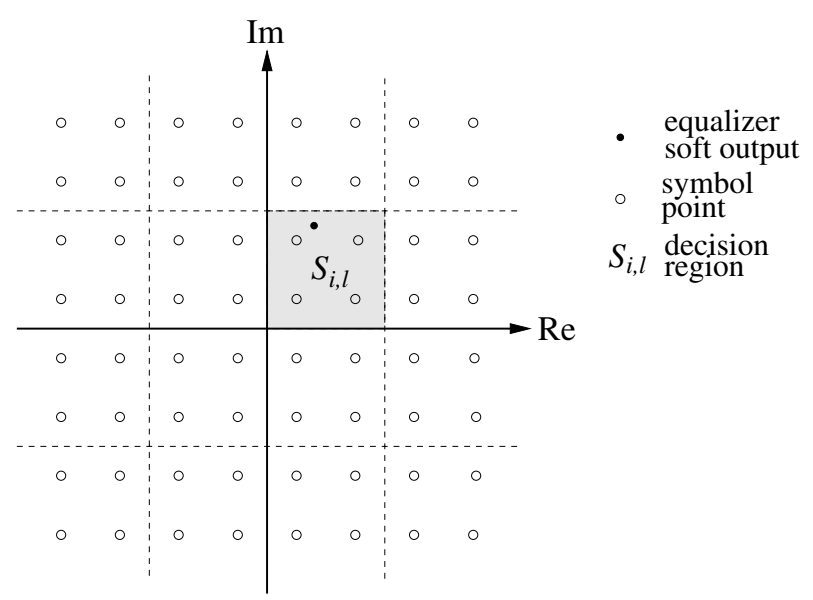

Fig. 3. Illustration of the local decision regions for the soft decision-directed adaptation procedure in a 64-QAM constellation. 
TABLE I

Comparison of the computational complexity per weight update. The number of receiver antennas is $L$ and the order of temporal filters is $2 m$.

\begin{tabular}{cccc}
\hline equalizer & multiplications & additions & $\exp (\bullet)$ \\
\hline CMA & $8 \times(L \times 2 m)+6$ & $8 \times(L \times 2 m)$ & - \\
CMA+DD & $16 \times(L \times 2 m)+8$ & $20 \times(L \times 2 m)$ & - \\
CMA+SDD & $12 \times(L \times 2 m)+29$ & $14 \times(L \times 2 m)+21$ & 4 \\
\hline
\end{tabular}

The complexity of the CMA aided SDD scheme is characterized in Table I. The four $\exp (\bullet)$ evaluations can be implemented using a look-up table in practice. The CMA aided DD scheme [8] can also be extended to the blind STE considered. The complexity of the CMA assisted DD blind STE as well as that of the pure CMA scheme was also listed in Table I.

\section{Simulation Study}

Time-invariant system. Two performance criteria were used for assessing the convergence rate of a blind STE. The first one was the decision-based estimated MSE calculated for each adaptation sample based on a block of $N_{\mathrm{MSE}}$ number of $T_{s}$-spaced data samples, yielding

$$
\mathrm{MSE}=\frac{1}{N_{\mathrm{MSE}}} \sum_{k=1}^{N_{\mathrm{MSE}}}|\mathcal{Q}[y(k)]-y(k)|^{2},
$$

where $\mathcal{Q}[\bullet]$ denotes the quantization operator. The second one was the maximum distortion (MD) measure defined as

$$
\mathrm{MD}=\frac{\sum_{i=0}^{N_{f}-1}\left|f_{i}\right|-\left|f_{i_{\max }}\right|}{\left|f_{i_{\max }}\right|}
$$

where $\mathbf{f}=\left[f_{0} f_{1} \cdots f_{N_{c}-1}\right]^{T}$ was the combined impulse response of the $L$ channels and the STE defined by

$$
\mathbf{f}=\sum_{l=1}^{L} \overline{\mathbf{w}}_{l}^{\mathrm{o}} \star \overline{\mathbf{a}}_{l}^{\mathrm{e}}+\overline{\mathbf{w}}_{l}^{\mathrm{e}} \star \overline{\mathbf{a}}_{l}^{\mathrm{o}},
$$

with $\star$ denoting convolution and $N_{f}=N_{c}+m-1$ being the length of the $T_{s}$-spaced combined impulse response, and in (17) we have $f_{i_{\max }}=\max \left\{f_{i}, 0 \leq i \leq N_{f}-1\right\}$. The equalizer's output phaser after convergence was also shown in Fig 5 using $N_{\text {test }}=6000 T_{s}$ spaced testing data samples not used during the adaptation stage.

In our simulations, $L=4$ receiver antennas were employed and 256-QAM was used. The $L=4 T_{s} / 2$-spaced channels were listed in Table II. The simulated CIRs were normalized according to $\overline{\mathbf{a}}_{l} /\left\|\overline{\mathbf{a}}_{l}\right\|$ for $1 \leq l \leq 4$ to ensure that each channel had a unit energy. The noise variance was $\sigma_{e}^{2}=0.00425$, yielding a SNR of $40 \mathrm{~dB}$. The length of the $T_{s} / 2$-spaced temporal filters was $2 m=10$. The learning curves of the two blind STEs, the

TABLE II

$T_{s} / 2$-spaced CIRs used in the simulated time-invariant SIMO system.

\begin{tabular}{ccccc}
\hline$l$ & \multicolumn{4}{c}{$\overline{\mathbf{a}}_{l}$} \\
\hline 1 & $-0.2+\mathrm{j} 0.3$ & $-0.5+\mathrm{j} 0.4$ & $0.7-\mathrm{j} 0.6$ & $0.2+\mathrm{j} 0.1$ \\
2 & $0.3+\mathrm{j} 0.2$ & $0.7-\mathrm{j} 0.5$ & $-0.5+\mathrm{j} 0.4$ & $0.4+\mathrm{j} 0.3$ \\
3 & $-0.1-\mathrm{j} 0.2$ & $0.6+\mathrm{j} 0.4$ & $-0.4+\mathrm{j} 0.3$ & $0.1+\mathrm{j} 0.3$ \\
4 & $0.6+\mathrm{j} 0.7$ & $0.5+\mathrm{j} 0.4$ & $-0.1-\mathrm{j} 0.2$ & $0.2+\mathrm{j} 0.1$ \\
\hline
\end{tabular}

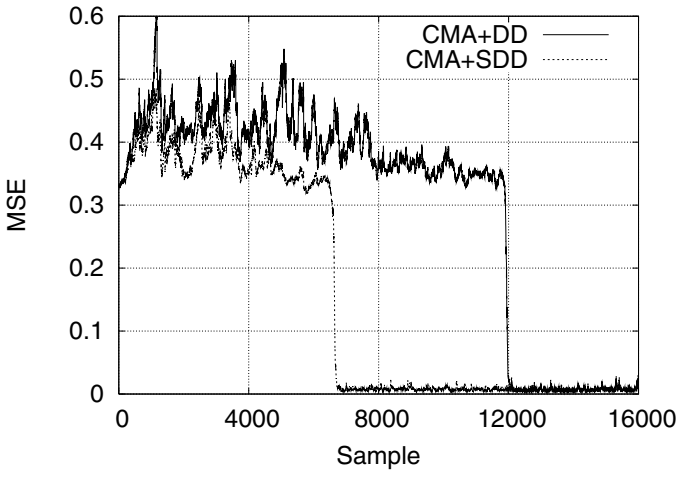

(a)

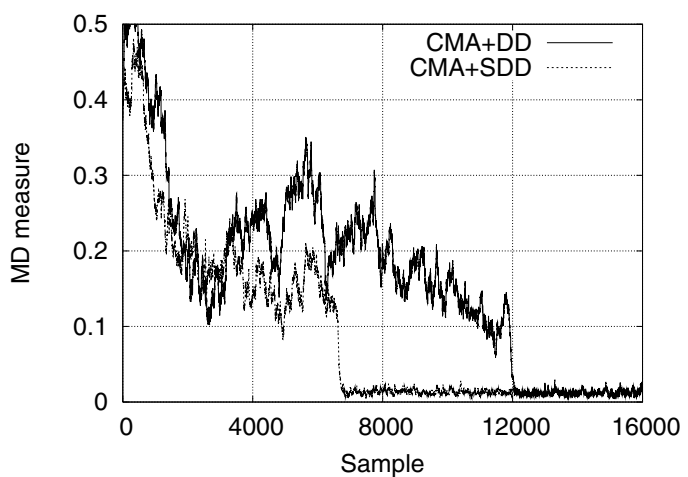

(b)

Fig. 4. Comparison of the convergence performance of the CMA+DD and CMA+SDD schemes in terms of (a) estimated MSE and (b) MD measure for the time-invariant CIR of Table II.

CMA+DD and CMA+SDD, in terms of the estimated MSE and MD measure, are depicted in Fig. 4 (a) and (b), respectively, while the equalizer's output signal constellations after convergence are illustrated in Fig. 5. It can be seen that both the CMA+DD and the CMA+SDD blind STEs had a similar steady-state equalization performance, but the latter had a significantly faster convergence.

Fading system. Again, $L=4$ receiver antennas were used, but 64-QAM was used at a symbol rate of 4 MBaud. The baseband continuous-time system was simulated. The transmission pulse had a raised-cosine characteristics with a roll-off factor 0.5 and a squareroot Nyquist characteristic at both the transmitter and receiver. The fading channels were implemented using the following tap-delay line models for $1 \leq l \leq 4$

$$
v_{l}(t)=c_{0, l}(t) u(t)+c_{1, l}(t) u\left(t-\tau_{1}\right)+c_{2, l}(t) u\left(t-\tau_{2}\right)
$$

where $u(t)$ was the transmitter output and $v_{l}(t)$ was the $l$ th fading channel output; $\tau_{1} \approx 0.44 T_{s}$ and $\tau_{2} \approx 1.13 T_{s}$; the magnitudes of the complex-valued tap weights $c_{i, l}(t), 0 \leq i \leq 2$, were i.i.d. Rayleigh processes and the root mean square (RMS) powers of $c_{i, l}(t)$ were $[0.7+j 0.70 .6+j 0.60 .5+j 0.5]$, for $0 \leq i \leq 2$, respectively. Continuously fluctuating fading was used at a fading rate of $40 \mathrm{~Hz}$. The noise power was $\sigma_{e}^{2}=0.011$. To simulate highly correlated SIMO channels that are commonly encountered in practice, the spatial covariance matrix generated for the scenario of a 
single transmit antenna to 4 receive antennas was represented by

$$
\mathbf{R}=\left[\begin{array}{cccc}
1 & \alpha & \beta & \gamma \\
\alpha^{*} & 1 & \alpha & \beta \\
\beta^{*} & \alpha^{*} & 1 & \alpha \\
\gamma^{*} & \beta^{*} & \alpha^{*} & 1
\end{array}\right]
$$

with $\alpha=0.97 \exp (-j 0.8), \beta=0.94 \exp (-j 1.6)$ and $\gamma=$ $0.88 \exp (-j 2.4)$ [14]. Let $\mathbf{R}=\mathbf{Q} \mathbf{\Sigma} \mathbf{Q}^{H}$, where $\mathbf{Q}$ consists of the eigenvectors associated with $\mathbf{R}$ and $\boldsymbol{\Sigma}$ is diagonal whose diagonal elements are the eigenvalues of $\mathbf{R}$. Then the receiver antenna outputs are represented by

$$
\left[\begin{array}{l}
x_{1}(t) \\
x_{2}(t) \\
x_{3}(t) \\
x_{4}(t)
\end{array}\right]=\mathbf{Q} \boldsymbol{\Sigma}^{1 / 2}\left[\begin{array}{l}
v_{1}(t) \\
v_{2}(t) \\
v_{3}(t) \\
v_{4}(t)
\end{array}\right]+\left[\begin{array}{l}
e_{1}(t) \\
e_{2}(t) \\
e_{3}(t) \\
e_{4}(t)
\end{array}\right] .
$$

The receiver outputs were sampled at the rate of twice the symbol rate and passed to a STE whose $T_{s} / 2$-spaced temporal filters had an order of 10 .

Fig. 6 depicts the CMA+DD STE's output signal constellations after (a) adaptation using 4000 symbols and (b) adaptation using 6000 symbols. Each signal constellation was shown for $6000 T_{s^{-}}$ spaced samples invoking continuous adaptation, since the channels were time-varying. Similarly, Fig. 7 shows the CMA+SDD STE's output signal constellations after (a) adaptation using 2500 symbols and (c) adaptation using 4500 symbols. It is readily seen that the CMA+SDD scheme had a significantly faster convergence rate than the CMA+DD arrangement.

\section{CONCLUSIONS}

In this paper, a novel low-complexity blind STE has been proposed for the SIMO system invoked by employing multiple receiver antennas, based on operating a CMA equalizer and a SDD equalizer concurrently. Simulation results have demonstrated that this blind STE performs well.

\section{REFERENCES}

[1] J.S. Blogh, L. Hanzo: Third-Generation Systems and Intelligent Wireless Networking - Smart Antennas and Adaptive Modulation, John Wiley, April 2002, ISBN 0-470-84519-8 430 pages.

[2] L. Hanzo, T.H. Liew, B.L. Yeap: Turbo Coding, Turbo Equalisation and SpaceTime Coding, John Wiley, August 2002, ISBN 0-470-84726-3, 766 pages.

[3] L. Hanzo, M. Münster, B.J. Choi and T. Keller: OFDM and MC-CDMA for Broadband Multi-user Communications, WLANs and Broadcasting, John Wiley - IEEE Press, July 2003, 980 pages.

[4] L. Hanzo, L-L. Yang, E-L. Kuan and K. Yen: Single- and Multi-Carrier DSCDMA: Multi-User Detection, Space-Time Spreading, Synchronisation, Standards and Networking, IEEE Press - John Wiley, August 2003, 1060 pages.

[5] A.J. Paulraj and B.C. Ng, "Space-time modems for wireless personal communications," IEEE Personal Communications, Vol.5, No.1, pp.36-48, 1998

[6] A. Paulraj, R. Nabar and D. Gore, Introduction to Space-Time Wireless Communications. Cambridge: Cambridge University Press, 2003.

[7] S. Chen, "Low complexity concurrent constant modulus algorithm and soft decision directed scheme for blind equalisation," IEE Proc. Vision, Image and Signal Processing, Vol.150, No.5, pp.312-320, 2003.

[8] F.C.C. De Castro, M.C.F. De Castro and D.S. Arantes, "Concurrent blind deconvolution for channel equalization," in Proc. ICC'2001 (Helsinki, Finland), June 11-15, 2001, Vol.2, pp.366-371.

[9] R. Johnson, Jr., P. Schniter, T.J. Endres, J.D. Behm, D.R. Brown and R.A. Casas, "Blind equalization using the constant modulus criterion: a review," Proc. IEEE, Vol.86, No.10, pp.1927-1950, 1998.

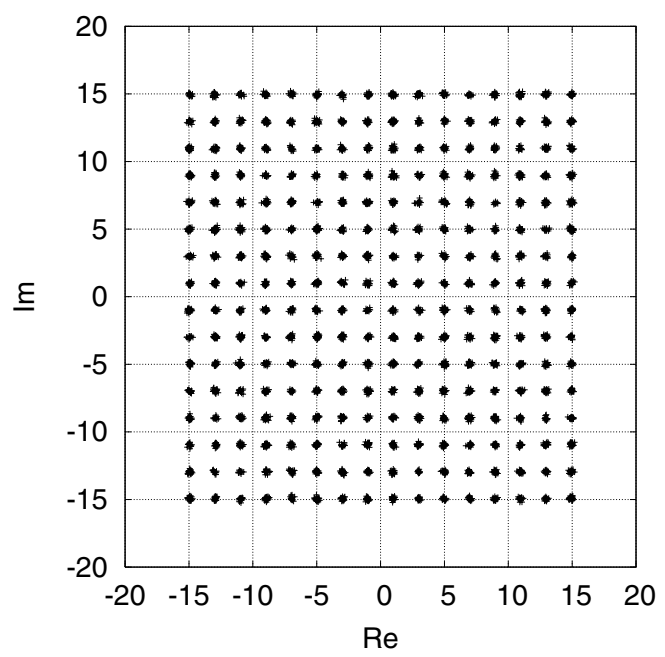

(a)

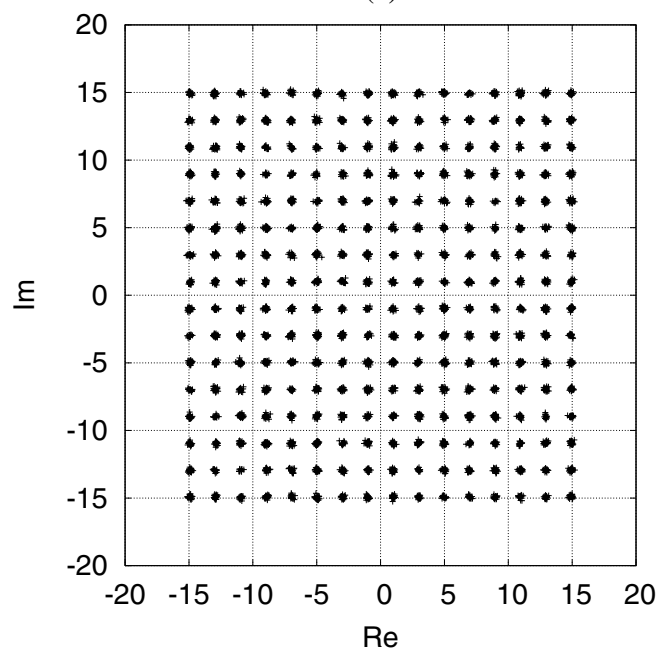

(b)

Fig. 5. Equalizer output signal constellations after convergence (a) the CMA+DD, and (b) CMA+SDD for the time-invariant CIR if Table II.

[10] D. Godard, "Self-recovering equalization and carrier tracking in two-dimensional data communication systems," IEEE Trans. Communications, Vol.COM-28, pp.1867-1875, 1980.

[11] J.R. Treichler and B.G. Agee, "A new approach to multipath correction of constant modulus signals," IEEE Trans. Acoustics, Speech and Signal Processing, Vol.ASSP-31, No.2, pp.459-472, 1983.

[12] S. Chen, S. McLaughlin, P.M. Grant and B. Mulgrew, "Reduced-complexity multi-stage blind clustering equaliser," in Proc. ICC'93 (Geneva, Switzerland), 1993, Vol.2, pp.1149-1153.

[13] S. Chen, S. McLaughlin, P.M. Grant and B. Mulgrew, "Multi-stage blind clustering equaliser," IEEE Trans. Communications, Vol.43, No.3, pp.701-705, 1995.

[14] 3GPP, "Tx diversity solutions for multiple antennas," 3GPP TSG RAN WG1 document TR25.869 V0.1.1, Nov. 2001. 


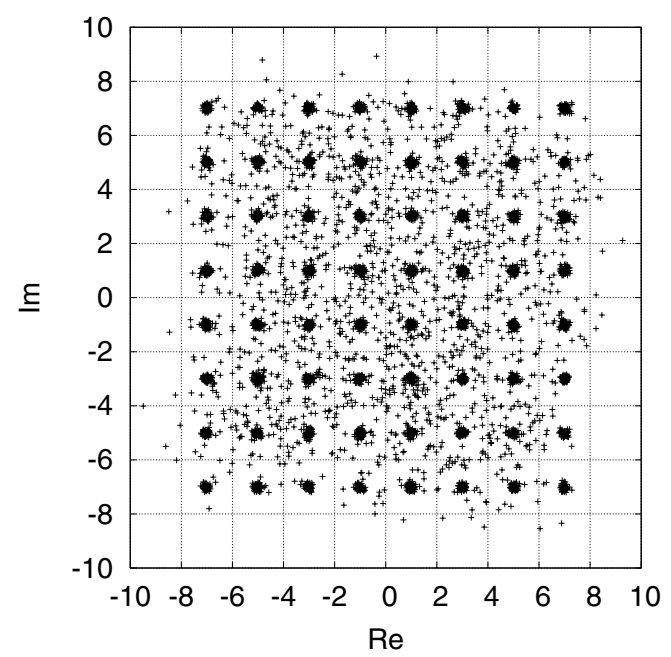

(a)

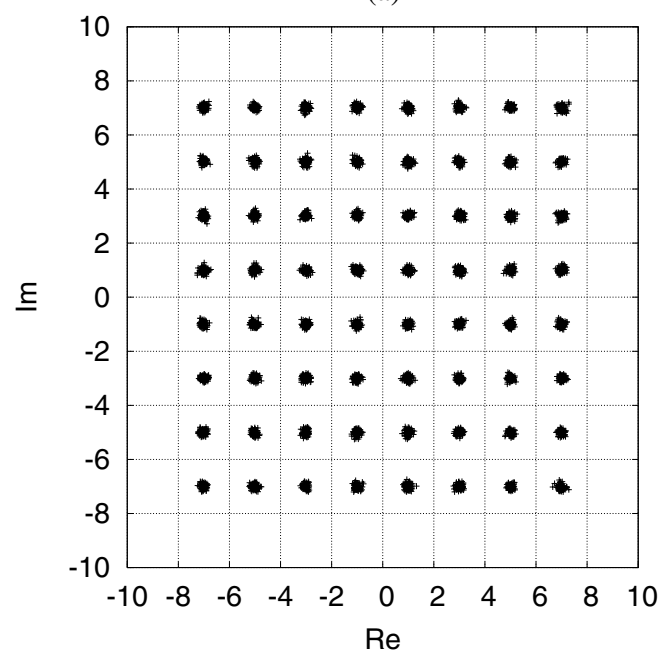

(b)

Fig. 6. CMA+DD equalizer output signal constellations for the fading system: (a) after adaptation of 4000 symbols and (b) after adaptation of 6000 symbols. 6000 $T_{s}$-spaced samples were used in showing the signal constellation with continuous adaptation.

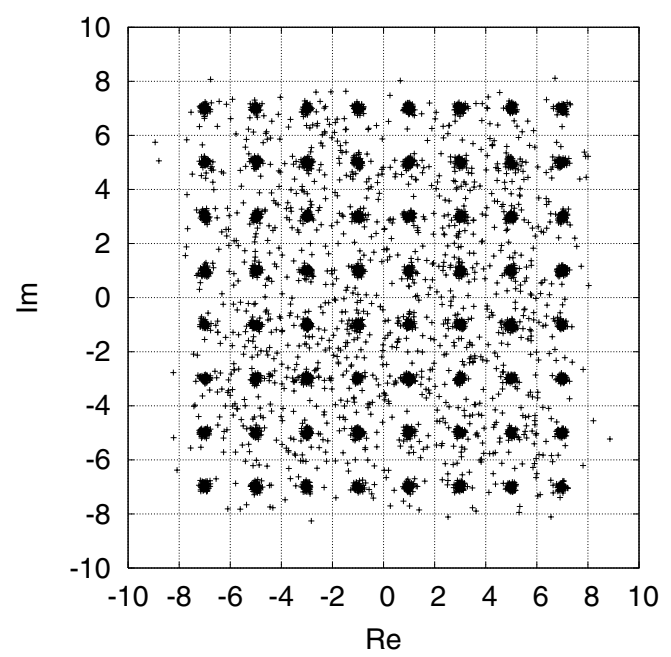

(a)

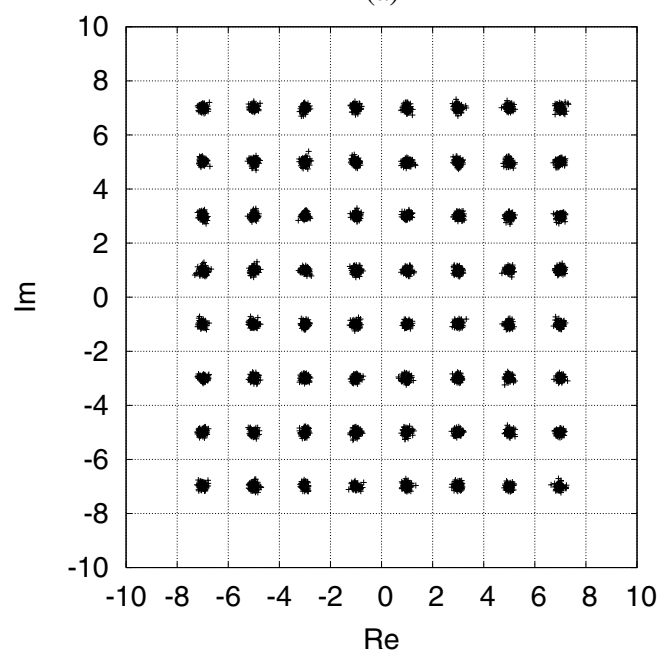

(b)

Fig. 7. CMA+SDD equalizer output signal constellations for the fading system: (a) after adaptation of 2500 symbols and (a) after adaptation of 4500 symbols. 6000 $T_{s}$-spaced samples were used in showing the signal constellation with continuous adaptation. 